\title{
Brain metastasis in renal cancer patients: metastatic pattern, tumour-associated macrophages and chemokine/chemoreceptor expression
}

\author{
L Wyler ${ }^{1}$, C U Napoli ${ }^{2}$, B Ingold ${ }^{1}$, T Sulser ${ }^{3}$, M Heikenwälder ${ }^{4}$, P Schraml ${ }^{1}$ and H Moch*,1 \\ ${ }^{1}$ Institute of Surgical Pathology, University Hospital Zurich, Zurich, Switzerland; ${ }^{2}$ Institute of Pathology, University Hospital Basel, \\ Basel, Switzerland; ${ }^{3}$ Clinic for Urology, University Hospital Zurich, Zurich, Switzerland and ${ }^{4}$ Institute of Virology, Technische \\ Universität München (TUM), Helmholtz Zentrum München (HMGU), München, Germany
}

Background: The mechanisms of brain metastasis in renal cell cancer (RCC) patients are poorly understood. Chemokine and chemokine receptor expression may contribute to the predilection of RCC for brain metastasis by recruitment of monocytes/ macrophages and by control or induction of vascular permeability of the blood-brain barrier.

Methods: Frequency and patterns of brain metastasis were determined in 246 patients with metastatic RCC at autopsy. Expression of CXCR4, CCL7 (MCP-3), CCR2 and CD68 ${ }^{+}$tumour-associated macrophages (TAMs) were analysed in a separate series of 333 primary RCC and in 48 brain metastases using immunohistochemistry.

Results: Fifteen percent of 246 patients with metastasising RCC had brain metastasis. High CXCR4 expression levels were found in primary RCC and brain metastases (85.7\% and $91.7 \%$, respectively). CCR2 $(52.1 \%)$ and CCL7 expression (75\%) in cancer cells of brain metastases was more frequent compared with primary tumours (15.5\% and $16.7 \%$, respectively; $P<0.0001$ each). The density of $\mathrm{CD}_{68}{ }^{+}$TAMs was similar in primary RCC and brain metastases. However, TAMs were more frequently CCR2-positive in brain metastases than in primary RCC $(P<0.001)$.

Conclusion: Our data demonstrate that the monocyte-specific chemokine CCL7 and its receptor CCR2 are expressed in tumour cells of RCC. We conclude that monocyte recruitment by CCR2 contributes to brain metastasis of RCC.

Brain metastasis is a relevant complication in the course of renal cancer progression because it is a major cause of morbidity and mortality in renal cancer patients. Metastasis to the brain has been reported in $2-16 \%$ of renal cancer patients (Gay et al, 1987; Schouten et al, 2002; Bianchi et al, 2012). This is a significant proportion of the advanced RCC patient population with an extremely unfavourable prognosis (Shuch et al, 2008).

Treatment of metastatic renal cancer is difficult because most RCCs are resistant to radio- and chemotherapy (Motzer et al, 1996). Chemo- and radiotherapy have also limited central nervous system (CNS) efficacy in brain metastases of renal cancer (Culine et al, 1998). Renal cancers are considered immunogenic tumours that are frequently infiltrated by immune cells (Gouttefangeas et al, 2007). Various immunotherapeutic strategies have been used for metastatic RCC. However, immunotherapy has played a limited role in patients with brain metastasis because the brain is considered an immune-privileged site. Recently, multikinase inhibitor therapy has shown promising results in patients with metastatic RCC. Sorafenib, an oral multikinase inhibitor, reduced the occurrence of brain metastases (Massard et al, 2010). Owing to a poor prognosis, patients with metastatic renal cancer in the CNS are often excluded from clinical trials with multikinase inhibitors 
(Medioni et al, 2007; Helgason et al, 2008; Thibault et al, 2008; Massard et al, 2010); however, they are frequently treated with multikinase inhibitors in clinical practice.

Despite the clinical significance of brain metastases and the importance of their detection for patient treatment selection, the metastatic pathways of renal cancer to the brain and how inflammatory mediators and inflammatory cells exactly contribute to brain metastases remain elusive (Nathoo et al, 2005). Different tendencies of metastasis to specific organs depend on intrinsic properties of the primary tumour and specific characteristics of the target organ. Such tumour features include chemokine/chemoreceptor expression in tumours and brain microvessels and/or inflammatory cells in the tumour microenvironment (Muller et al, 2001; Pan et al, 2006a). For brain metastasis, blood-brain barrier (BBB) disruption with subsequent increased vascular permeability and leucocyte migration into the brain is pivotal. Some recent studies indicate that migration of inflammatory cells, including tumour-associated macrophages (TAMs) may contribute to the persistence of increased vascular permeability (de Vries et al, 2006; Doolittle et al, 2007; Colotta et al, 2009). Such TAMs are potentially recruited to tumours through specific chemokine/ chemokine receptor interactions.

Interactions of chemokines with their receptors have a role in homing of neoplastic cells from the primary site to the target organ and in local progression of the metastasis by inducing angiogenesis (Liang et al, 2004; Andre et al, 2006; Pan et al, 2006b). Members of the chemokine family include CC and CXC chemokines. CC chemokines induce the migration of monocytes to leave the bloodstream and enter the surrounding tissue to become tissue macrophages. Examples for CC chemokines include monocyte chemo/attractant protein-1 (MCP-1, also termed CCL2) and the closely related monocyte-specific chemokine 3 (MCP-3, also termed CCL7). CC chemokines bind to CC chemokine receptors (CCRs) that are integral membrane proteins of lymphocytes and macrophages. CCL2 and CCL7 and their pivotal receptors CCR1, 2 and 3 were shown to have a role in inflammatory processes in the brain (McCandless et al, 2008a, b). Moreover, increased expression of CCR2 ligands by tumour cells correlates with enhanced metastasis, poor prognosis of various tumours (e.g. prostate cancer, breast cancer, colon cancer and cervix cancer) and recruitment of inflammatory $\mathrm{CCR}^{+}$monocytes (Zijlmans et al, 2006; Yoshidome et al, 2009; Zhang et al, 2010a; Soria et al, 2011). Using a mouse model, we have recently demonstrated that colon carcinoma-derived CCL2 attracts CCR2 ${ }^{+}$monocytes and at the same time also activates endothelial cells through CCR2 (Wolf et al, 2012). Activation of endothelial cells through CCR2 induces vascular permeability, enabling efficient tumour cell extravasation and metastases formation.

In contrast, CXC chemokines specifically induce the migration of neutrophils and bind to CXC chemokine receptors. In renal cancer, the CXCR4/CXCL12 system is most important because CXCR4 is regulated by VHL/HIF (Staller et al, 2003). CXCR4 expression was identified as a prognostic factor in breast (Andre et $a l, 2006$ ) and renal cell cancer (RCC) (Staller et al, 2003; D'Alterio et al, 2010a). The CXCR4/CXCL12 axis also has a relevant role in experimental models of metastasis (Stamatovic et al, 2005; Wang et al, 2010) and is supposed to be crucial in brain metastases formation from breast cancer. CXCR4 and its ligand, CXCL12, are expressed in astrocytes, microglia, neurons and endothelial cells of the brain (Lazarini et al, 2003). Experimental data have shown that inhibition of the CXCR4/CXCL12 receptor/ ligand interaction may decrease metastasis establishment and progression within the brain, as well as migration of tumour cells across an in vitro model of BBB (Phillips et al, 2003; Lee et al, 2004; Pan et al, 2006b; Salmaggi et al, 2009).

To further understand the mechanisms of brain metastasis in renal cancer, we evaluated patterns of metastasis in a large number of autopsies. In addition, the relevance of TAMs and the expression patterns of selected chemokines/chemokine receptors in brain metastases and in primary clear cell RCCs were investigated. We demonstrate that brain metastasis occurs only in a subgroup of patients with metastatic RCC. CCR2-positive TAMs as well as tumour-cell-derived CCL7 and-CCR2 are shown to be relevant for brain metastasis in RCC patients.

\section{MATERIALS AND METHODS}

Autopsy. In a consecutive series of $40^{\prime} 021$ autopsies performed at the Institute of Pathology of the University of Basel between 1967 and 1995, there were 871 epithelial renal tumours with a diameter $>5 \mathrm{~mm}$. Autopsies were performed according to a standardised protocol based on modifications of the Virchow method (Bubendorf et al, 2000). According to Swiss law between 1967 and 1995, consent from the deceased prior to death or the next of kin was tried to obtain by the clinicians. The organs were macroscopically investigated for the presence or absence of metastases. All lesions in parenchymal organs suspicious for a metastasis were microscopically analysed. The vertebral column was also carefully screened for metastases macroscopically and suspicious lesions were microscopically evaluated. Other bones were not routinely investigated. Autopsy of the brain was performed in all cases. If there was no clinical evidence for metastasis, $2-\mathrm{cm}$ thick brain slices were macroscopically analysed for presence or absence of brain metastases. If there was clinical evidence for metastasis, the brain, the cerebellum and the brainstem were cut after fixation in $1-\mathrm{cm}$ intervals and displayed for examination and microscopic sampling.

Four hundred and seventy-five renal cell carcinomas had a diameter $>1 \mathrm{~cm}$ and 161 autopsies had a status after nephrectomy. The mean tumour diameter was $3.9 \pm 4 \mathrm{~cm}$ (median $2 \mathrm{~cm}$ ). The average age of tumour patients at autopsy was $71.9 \pm 11$ years. The mean age of patients after nephrectomy was significantly higher $(73 \pm 11$ years) than in patients without nephrectomy $(67.7 \pm 10.8$ years; $P<0.001)$. Renal cancer was more common in males than in females (63\% vs $37 \%)$. There was no difference between the left and the right kidney. Tumour stage and maximum tumour diameter had been assessed macroscopically in most tumours. If not indicated, the stage according to the TNM classification was reconstructed from the tumour extension described in the autopsy reports. Sixty-seven percent of the cases were pT 1 and pT 2 and only $23 \%$ had pT3 and pT4 stages. In $8.8 \%$, the tumour stage could not be assessed retrospectively.

Of these 636 patients, metastases were observed in 246 patients. A schematic overview of the whole process is given in Figure 1. Presence of metastasis was significantly associated with tumour size $(P<0.0001)$. The following metastatic sites were identified according to the data in the autopsy reports: lung, bone and bone marrow, lymph node (regionary, juxtaregionary), liver, adrenal gland, brain, retroperitoneum and peritoneum, soft tissue, skin, retina, gastrointestinal tract, pancreas, breast, spleen, gall bladder, parotid, contra lateral kidney, thyroid, genital system (testis, vagina, ovary) and urinary bladder. For the purpose of the analysis, metastatic sites were then categorised as lymphatic metastasis, which includes regional and juxtaregional regions, as well as haematogenous metastasis. Brain and bone metastases were considered independently. The evaluation was also separately performed in patients with and without nephrectomy.

Surgical tumourectomies and biopsies of brain metastases. As tissue samples form the autopsies described above were not available and not useful for immunohistochemical studies, we used two previously described TMAs with formalin-fixed, paraffinembedded tissue samples of 333 primary RCC and 54 renal cancer 


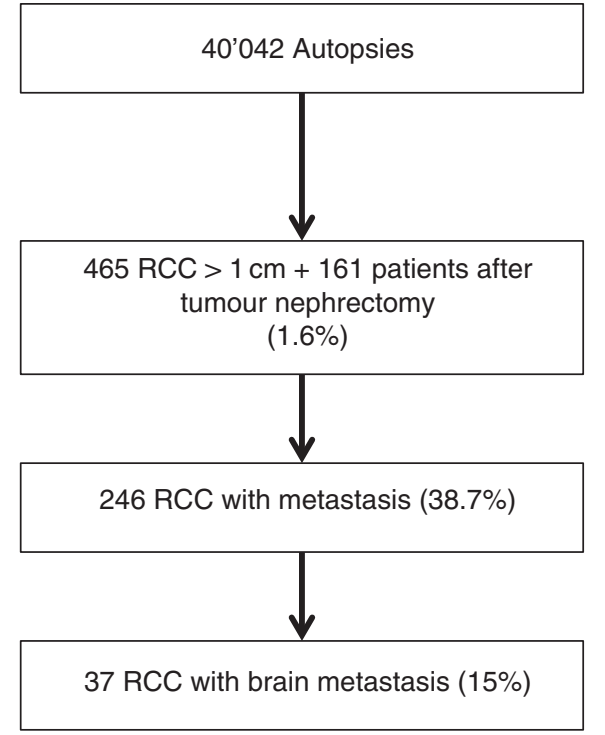

Figure 1. Schematic flow with total numbers of RCC, metastasising RCC and brain metastasis RCC in $40^{\prime} 042$ autopsies performed at the University of Basel.

\begin{tabular}{|c|c|c|c|c|c|}
\hline & $\begin{array}{c}\text { Age } \\
\text { (years) }\end{array}$ & Gender & $\begin{array}{c}\text { Grade } \\
\text { (Fuhrman) }\end{array}$ & $\begin{array}{l}\text { Tumour } \\
\text { stage }\end{array}$ & $\begin{array}{l}\text { Survival } \\
\text { (months) }\end{array}$ \\
\hline Patient 1 & 58 & Male & 4 & $3 a$ & 9 \\
\hline Patient 2 & 65 & Male & 3 & 2 & 36 \\
\hline Patient 3 & 57 & Male & 3 & $1 b$ & 34 \\
\hline Patient 4 & 66 & Male & 3 & 2 & $97^{a}$ \\
\hline Patient 5 & 57 & Male & 4 & $3 b$ & 7 \\
\hline Patient 6 & 64 & Male & 3 & 3 & 44 \\
\hline Patient 7 & 68 & Male & 3 & $1 a$ & 37 \\
\hline Patient 8 & 63 & Male & 3 & $1 \mathrm{~b}$ & $55^{a}$ \\
\hline Patient 9 & 50 & Male & 3 & 2 & $49^{a}$ \\
\hline Patient 10 & 46 & Male & 3 & 2 & NA \\
\hline \multicolumn{6}{|c|}{$\begin{array}{l}\text { Abbreviations: } N A=\text { not available; } R C C=\text { renal cell cancer. } \\
\text { a Alive at this time point. }\end{array}$} \\
\hline
\end{tabular}

brain metastases as described previously (Ingold et al, 2008, 2009; Dahinden et al, 2010). The primary tumours were derived from the Institute of Surgical Pathology, University of Zurich (1993-2003), and the brain metastases were from the Institute of Neuropathology, University of Zurich (1981-2005). For 10 of 48 brain metastases, matched paired primary RCC specimens were also available. The clinicopathological data are listed in Table 1. All tumour samples have been re-evaluated systematically by one pathologist (HM). This project has been approved by the local ethics committee (ref. number StV 37-2005).

Immunohistochemistry. Sections of TMA $(2.5 \mu \mathrm{m})$ on glass slides were subjected to immunohistochemical analysis according to the Ventana (Tucson, AZ, USA) and Bond (Vision BioSystems, Newcastle Upon Tyne, UK) automat protocols. CXCR4 (dilution $1: 1200$; mouse monoclonal; clone 12G5; Zymed Laboratories, Inc., San Francisco, CA, USA), CCL7 (dilution 1:500; rabbit polyclonal, GenWay Biotech, Inc., San Diego, CA, USA), CCR2 (dilution $1: 200$; rabbit monoclonal; clone E68; Abcam Limited, Cambridge,
UK), CD68 (dilution 1:50; mouse monoclonal; clone PG-M1; Dako, Glostrup, Denmark), CCL2 (dilution 1:100; mouse monoclonal; clone 23002; R\&D Systems, Minneapolis, MN, USA) stainings were performed and analysed under a Leitz Aristoplan microscope (Leica, Wetzlar, Germany).

Cell lines with known CXCR4 and CCL7 expression were used as positive controls. Staining intensity of tumour cells and endothelial cells was scored negative $(0)$, moderate $(+1)$ or strong $(+2)$. In contrast, the density of immunostained macrophages (CD68 and CCR2) was scored as 0 (no infiltrate; $0-1$ macrophage per spot), +1 (sparse infiltrate; 2-20 macrophages per spot), +2 (loose infiltrate; $21-40$ macrophages per spot) and +3 (dense infiltrate; $>40$ macrophages per spot). There was no evaluation of the CCR2 staining intensity in positive macrophages as staining was either strong or absent similar to CD68 staining.

Statistical analysis. Analyses of variants (ANOVAs) were performed to analyse the relationship of nominal parameters with continuous variables (e.g., patient age, tumour diameter). Contingency table analysis and paired sample $t$-tests were used for the analysis of the associations between protein expression frequencies in primary RCC and brain metastases. SPSS 18.0 software (IBM Schweiz AG, Zurich, Switzerland) was used for the calculations.

\section{RESULTS}

Brain metastases at autopsy. Lymph node metastasis was observed in $23 \%$. The most common sites of haematogenous metastasis were lung (75\%), liver and bone (40\%), soft tissue $(34 \%)$, adrenal gland $(22 \%)$, pleura $(31 \%)$ and heart/pericard (16\%). Brain metastases were seen in $15 \%$ of all RCC patients with metastasis (see Figure 1) and in $18.6 \%$ of patients with lung metastases. Presence or absence of brain metastasis was not dependent on the size of the primary tumour; however, brain metastases were not found in patients with primary tumours $\leqslant 3 \mathrm{~cm}$. In most cases, lung metastasis coincided with metastasis in the brain. Only in one patient, brain metastasis was found in the absence of detectable lung metastasis.

Renal cancer is thought to metastasise according to the cavatype of metastasis through the lung. To analyse the existence of alternative pathways, we categorised patients into presence or absence of lung metastasis. Interestingly, 61 patients (25\%) showed metastasis without lung metastasis. Sixty-eight patients had concomitant lung and other haematogenous metastases, whereas only lung metastasis was observed in $8 \%$ of patients. This distribution was not different in patients with or without nephrectomy. Almost all cases with lymphatic spread showed evidence of haematogenous metastases (92\%). Interestingly, there were 154 tumours between 1 and $2 \mathrm{~cm}$ in diameter. In these cases, two patients $(1.3 \%)$ showed evidence of metastasis.

Despite the strong association between tumour stage and haematogenous metastasis, the capability of a tumour to cause metastatic spread is obviously not only defined by its local stage or diameter. Therefore, we screened for non-metastatic cases in patients with clear-cut venous invasion. In 99 cases, information about the macroscopical existence of a tumour thrombus in the vena renalis $(80 \%)$ or in the vena cava $(20 \%)$ could be retrieved. In $73 \%$ of these cases, there were haematogenous metastases, meaning that $27 \%$ with tumour thrombus in the vena cava showed no evidence of haematogenous metastasis. This phenomenon has been described as 'metastatic inefficiency' (Glaves et al, 1988; Weiss et al, 1988).

CXCR4 and CCL7 expression in primary renal cancer and brain metastases. As the brain metastases derived exclusively from clear cell RCCs, only this tumour subtype $(n=245)$ was compared with 
A
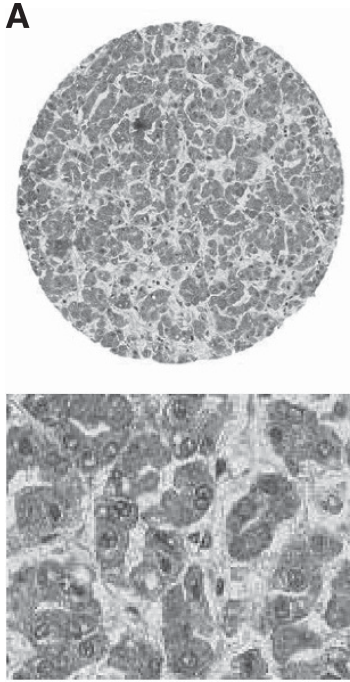

B
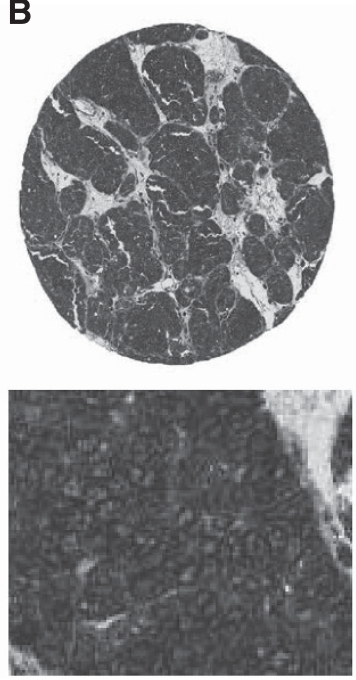

C
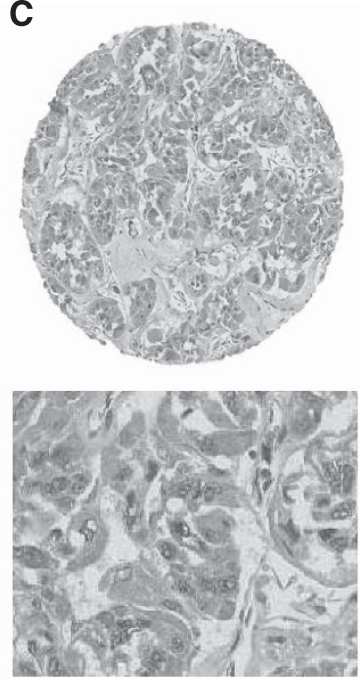

Figure 2. Moderate and strong expression of CXCR4 (A, B) and CCL7 (C) in RCC. Upper panel: TMA spots (magnification $\times 4$ ); lower panel: magnified spot areas $(\times 40)$.

the brain metastases from which 48 were analysable. A moderate to strong expression of CXCR4 was seen with similar frequencies in primary RCC and RCC brain metastases $(85.7 \%$ and $91.7 \%$, respectively). In primary $\mathrm{RCC}$, there was a correlation between CXCR4 expression and higher Fuhrman grade $(P<0.05)$.

CCL7 showed an increased positivity in tumour cells of RCC brain metastasis compared with primary tumours (75\% vs $16.7 \%$; $P<0.0001)$. A similar result was obtained from the 10 paired primary and brain metastasis samples ( $80 \%$ vs $20 \%$; $P<0.01)$. Examples of CXCR4- and CCL7-expressing tumours are illustrated in Figure 2. CCL7 positivity in stromal and endothelial cells showed no expression differences in primary RCC and brain metastases (data not shown), and all endothelial cells in brain metastasis showed either a moderate $(22.5 \%)$ or strong $(77.5 \%)$ CCL7 expression. The data are illustrated in Figure 3. CCL2 could not be analysed because CCL2 antibody gave no reliable immunohistochemical staining results in formalin-fixed, paraffin-embedded tumours.

Tumour-associated macrophages. There was sparse to dense macrophage infiltrate inside vital tumour parenchyma in almost all primary RCC. Only in two of the 245 primary RCCs, we could not identify CD68-positive macrophages. In primary RCC, a dense CD68positive macrophage infiltrate was associated with higher Fuhrman grade $(P<0.0023)$. The $\mathrm{CD}^{+} 8^{+}$macrophage score was comparable in primary RCC and brain metastases (dense infiltration $50.6 \%$ vs $56.3 \%$ ). In a more detailed analysis, TAMs were analysed in primary RCC and brain metastases for CCR2 expression using a consecutive TMA section. Interestingly, CD $68^{+}$TAMs had significantly more frequently a dense CCR2-positive infiltrate in brain metastases compared with primary RCC $(13.9 \%$ vs $43.7 \%$; $P<0.001)$. Furthermore, we have found a more frequent CCR2 overexpression in tumour cells of brain metastases compared with primary tumour cells $(P<0.0001)$. The data, including those of the 10 matched paired samples, are shown in Figure 4. Examples of $\mathrm{CD}^{+} 8^{+}$and infiltrating macrophages as well as CCR2-expressing tumours are shown in Figure 5. Both findings support an involvement of CCR2 in RCCs metastasising to the brain.

\section{DISCUSSION}

Schouten et al (2002) demonstrated in a cohort of patients with colorectal, lung, breast and kidney cancer or with melanoma that the frequency of brain metastasis is highest in patients with lung and renal cancer. The percentage of brain metastases contributed by RCC is therefore greater than that expected from the frequency of this carcinoma among all carcinomas. This raises the two possibilities: first, that tumour cells from RCC are better able to reach or to survive in the brain than tumour cells from other cancers, or second, that one or more routes for dissemination of metastases to the brain may be available for RCC but are not readily available for other carcinomas.

In our study, we analysed the dissemination of renal cancer metastases in autopsies and studied cytokines and chemokines, which are potentially involved in the multistep process of metastasis. Our data provide novel evidence that monocyte recruitment by CCL7 and CCR2 may contribute to brain metastasis of renal cancer.

To determine brain metastasis in renal cancer, we first analysed autopsy results because autopsies offer an opportunity to study the distribution and frequency of metastases in different organ sites in a very late stage of tumour disease. Most frequent metastasis was seen in the lung. This high frequency of lung metastasis in RCC patients is consistent with the model that renal cancer metastasises primarily to the lung because all caval blood from the renal veins flows to the lungs. Interestingly, there was no evidence of lung metastasis in $25 \%$ of autopsies with metastatic RCC. Therefore, alternative metastatic pathways may exist for haematogenous renal cancer progression-for example, a backward paravertebral venous spread to the spine and the brain, which is relevant for prostate cancer (Bubendorf et al, 2000). However, our autopsy data also suggest a minor importance of this pathway for brain metastases of RCC because most brain metastases occurred in combination with lung metastases.

Compared with other metastatic sites, brain metastasis was less frequent. This is consistent with previous data from Bianchi et al (2012), who abstracted data from the Nationwide Inpatient Sample (NIS), an observational retrospective database relying on ICD-9 codes in the USA. Bianchi et al (2012) reported exclusive brain metastasis in only $2 \%$ among 11157 patients with metastatic RCC.

In other previous clinical, radiological or epidemiological studies, brain metastasis was reported in $2-17 \%$ of RCC patients (Gay et al, 1987; Schouten et al, 2002; Bianchi et al, 2012). The NIS data by Bianchi et al (2012) revealed brain metastasis in 16\% of patients with thoracic and concomitant bone metastases. Among our patients with lung metastases at autopsy, the rate of 

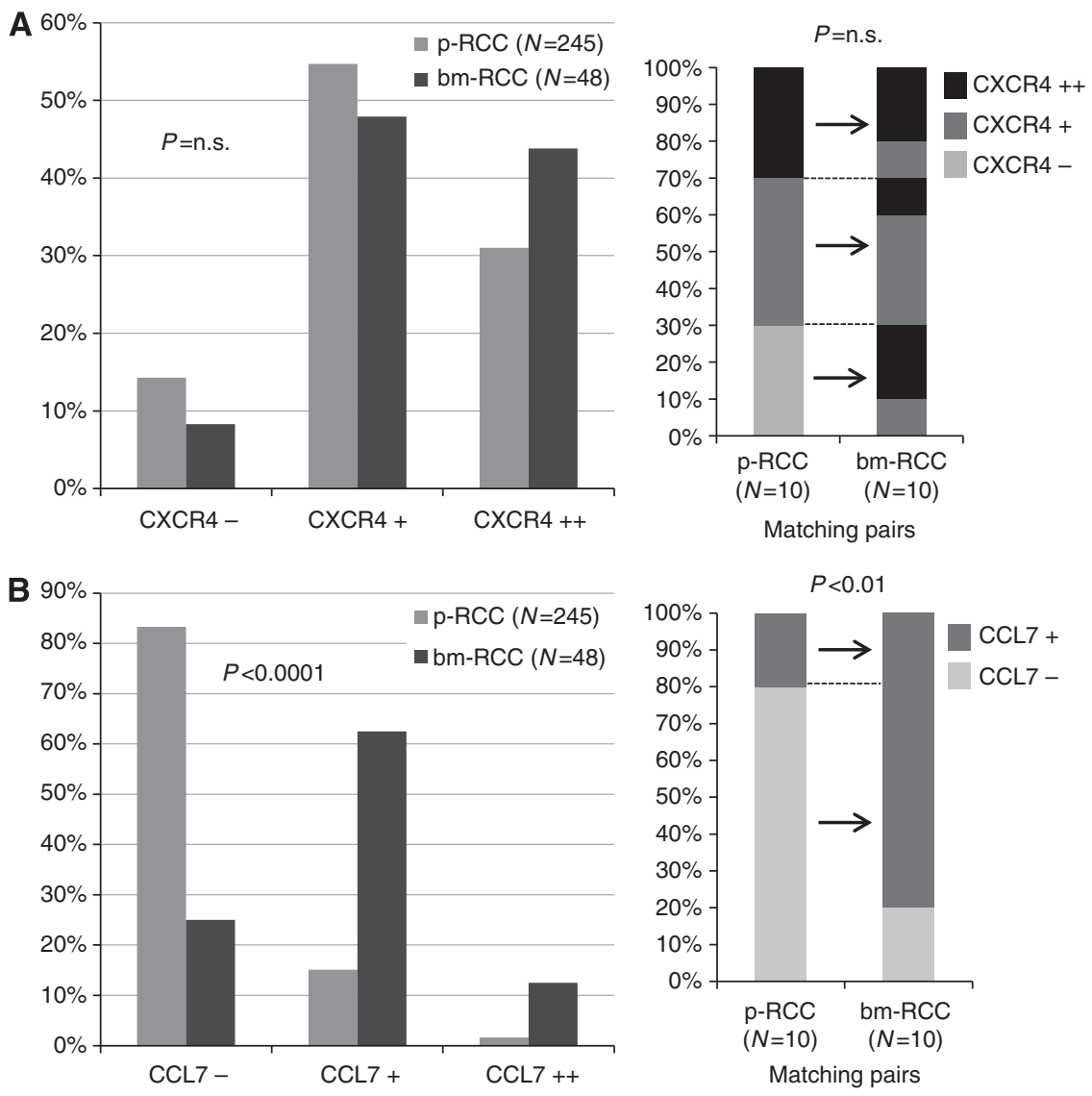

Figure 3. Expression frequencies of CXCR4 (A) and CCL7 (B) in primary RCC and RCC brain metastasis. Data of matched paired primary and brain metastasis RCC are shown on the right side. p-RCC: primary RCC; bm-RCC: brain metastasis RCC. Negative, moderate and strong expression levels are indicated with $(-),(+)$ and $(++)$, respectively.

brain metastasis was $18.6 \%$. There was only one patient with brain metastasis in the absence of lung metastasis. Given the fact that the brain is among the best-perfused organs of the body, these rates are comparatively low (Eichler and Loeffler, 2007; Eichler et al, 2011).

Taken together, all epidemiological and autopsy data suggest a very low risk for brain metastasis in the absence of lung metastases and a negligible relevance of a backward spread along paravertebral veins (Batson's plexus). Patients with brain metastasis have almost always concurrent metastases at other sites, confirming that this patient group represents a particularly unfavourable subset of individuals with very aggressive tumours (Gay et al, 1987; Schouten et al, 2002; Nathoo et al, 2004; Shuch et al, 2008).

The lung is effective in filtering out the majority of embolic renal cancer cells transported to it by blood from the right heart. Some renal cancer cells may pass via the pulmonary capillaries into the arterial circulation and produce brain metastases. In addition, the embolic malignant cells have to pass the $\mathrm{BBB}$. The predilection of RCC for brain metastasis is unclear. Specific characteristics of the tumour cells, cytokines and chemokines but also the tumour cell microenvironment in the brain are potential explanations for this 'homing' from kidney via lung to the brain. The tumour cell microenvironment is composed of endothelial cells, microglia and leukocytes. Stromal cells, infiltrating macrophages and tumour cells themselves were identified as sources of cytokines and chemokines, both at primary tumours and metastatic sites (Laubli and Borsig, 2010; Mishra et al, 2011).

To evaluate the role of different chemokines/chemoreceptors, we investigated CXCR4 expression in primary RCC and brain metastasis because CXCR4 is upregulated because of VHL inactivation in most cases of the clear cell renal cancer subtype (Staller et al, 2003). Various previous studies have confirmed that CXCR4 expression is associated with metastasis and/or prognosis of RCC (Staller et al, 2003; Pan et al, 2006b; D'Alterio et al, 2010b; Gahan et al, 2012). In this study, we have used a novel antibody with only membrane localisation (Fischer et al, 2008) as the relevance of a nuclear CXCR4 expression is controversial. It has been suggested that only cytoplasmic expression may reflect an active form of the receptor. Previous studies observed CXCR4 expression in $\sim 60-80 \%$ of RCC samples (Pan et al, 2006b; Salmaggi et al, 2009; D'Alterio et al, 2010b; Gahan et al, 2012), independent of the antibody, the immunohistochemical protocols or the metastatic localisation, arguing for a crucial role of CXCR4 for brain metastasis as well as for extra-CNS metastases. Our analysis identified CXCR4 expression in most RCC brain metastases. This is consistent with data from Salmaggi et al (2009) who reported a nuclear CXCR4 localisation in all brain metastases from breast $(n=18)$, lung $(n=17)$, kidney $(n=8)$, colorectal $(n=5)$ and other tumours $(n=8)$. The frequent CXCR4 expression in brain metastases is suggestive for a pivotal role of the CXCL12/CXCR4 axis for renal cancer metastasis to the brain. The predilection of RCC for metastasis to the brain cannot be explained by CXCR4 expression level alone, as we have also observed high CXCR4 expression levels in primary RCC.

Increased vascular permeability with subsequent $\mathrm{BBB}$ disruption is another prerequisite for brain metastasis. Recently, we have demonstrated that tumour-cell-derived CCL2 activates CCR2positive endothelium to increase vascular permeability in vivo (Wolf et al, 2012). The herewith presented study identified strong endothelial CCR2 expression in brain metastases but also stronger 

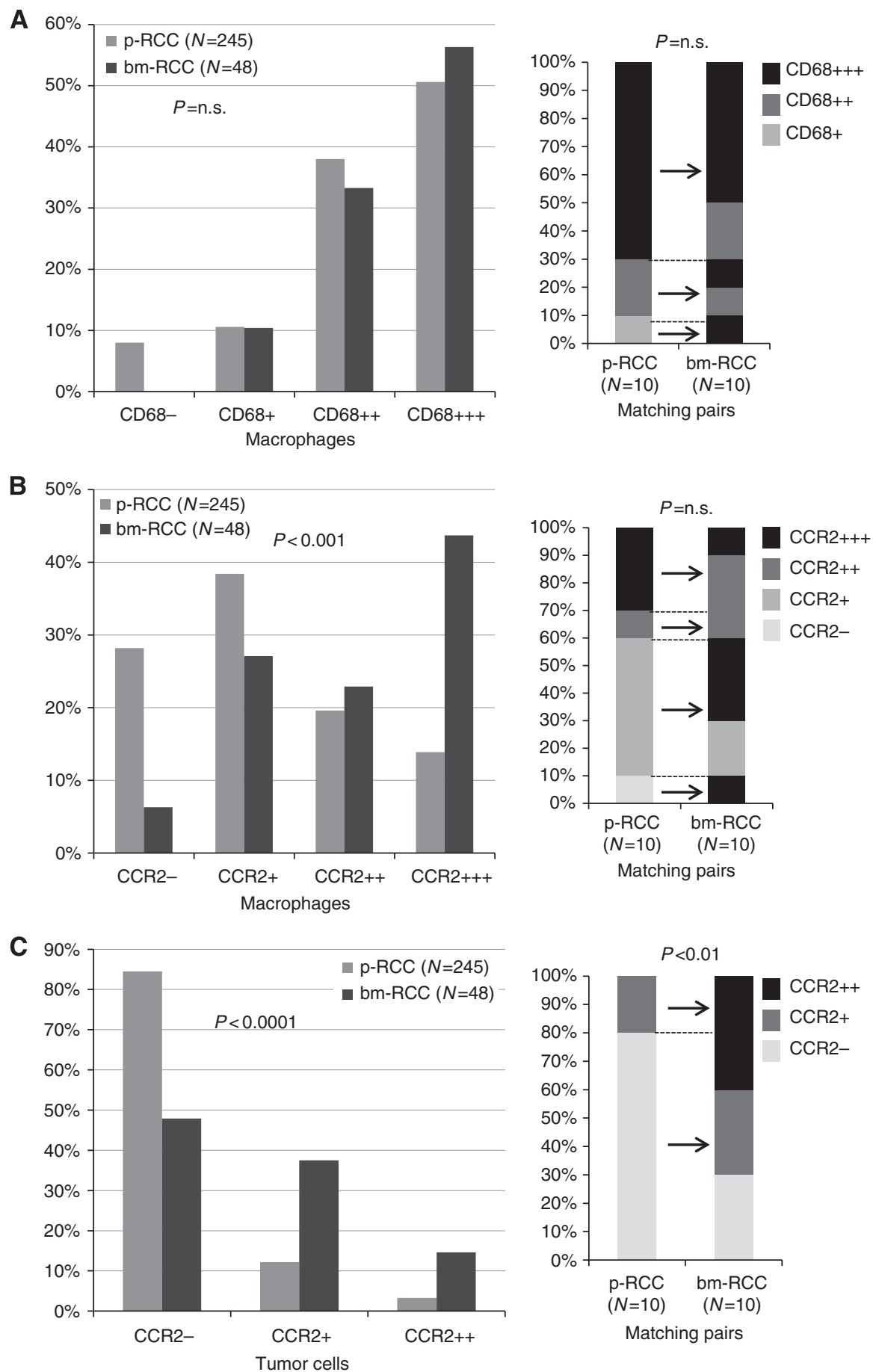

Figure 4. Infiltration density of CD68 (A) and CCR2 (B) positive tumour-associated macrophages in primary RCC and RCC brain metastasis. p-RCC: primary RCC; bm-RCC: brain metastasis RCC. No, sparse, loose and dense infiltrates of TAMs are indicated with $(-),(+),(++)$ and $(+++)$, respectively. (C) Expression frequency of CCR2 in tumour cells of primary RCC and RCC brain metastasis. Negative, moderate and strong expression levels are indicated with $(-),(+)$ and $(++)$, respectively. Data of matched paired primary and brain metastasis RCC are shown on the right side.

CCR2 expression in tumour cells of brain metastases compared with primary RCC. Whereas the strong endothelial CCR2 expression in brain metastases is consistent with the abovementioned model in colorectal cancer, the biological significance of CCR2 expression in tumour cells remains to be determined.

Recently, the density of different subpopulations of macrophages has been identified as a relevant prognostic marker in RCC (Dannenmann et al, 2013). In our study, we detected significantly more frequent CCR2-positive TAMs in brain metastases compared with primary RCC, whereas the degree of CD68-positive macrophages was similar in primary tumours and brain metastases. The pan-macrophage marker CD68 recognises all subsets of this heterogeneous cell type, including microglia as an integral part of the mononuclear phagocyte population in the CNS. Our data are consistent with a major role of CCR2-positive monocytes for brain metastasis.

It has been recently shown that myeloid-derived monocytes/ macrophages facilitate tumour cell extravasation and metastatic outgrowth in vivo (Qian et al, 2011) and that circulating CCR2positive monocytes are preferentially recruited to an injured brain 

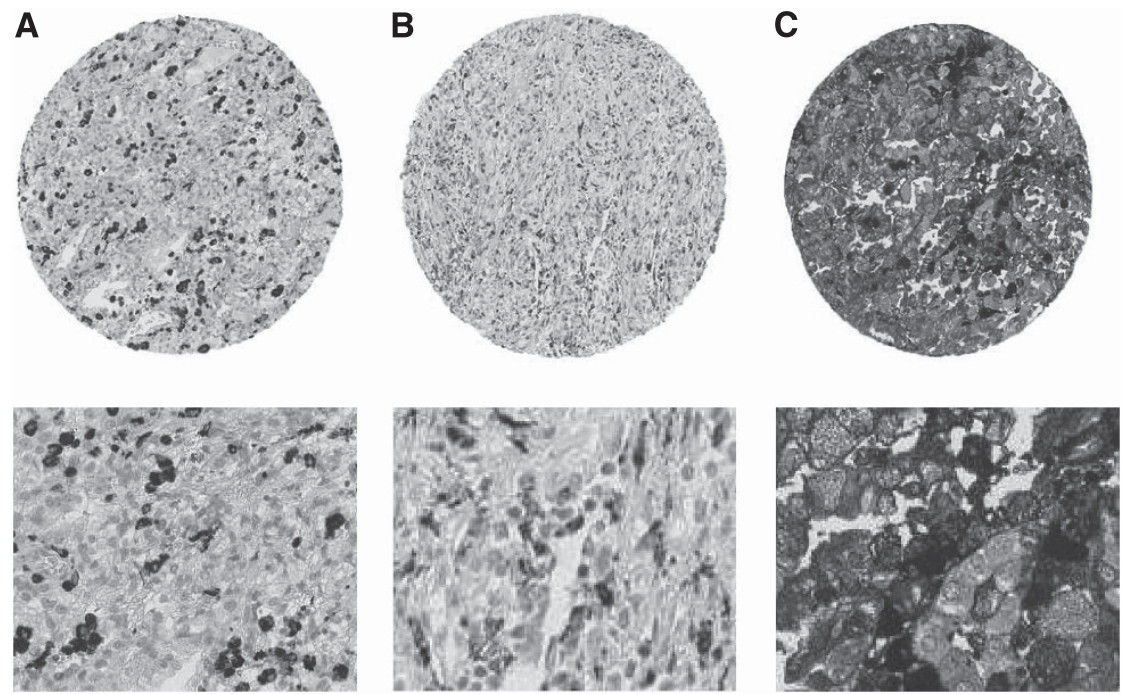

Figure 5. Dense infiltration of CD68- and CCR2-positive tumour-associated macrophages in RCC (A, B (tumour CCR2-negative)); moderate and strong expression of CCR2 $(\mathbf{C})$ in RCC cells. Upper panel: TMA spots (magnification $\times 4$ ); lower panel: magnified spot areas $(\times 40)$.

with further differentiation into microglia (Mildner et al, 2007). Both mechanisms can explain the significant higher number of CCR2-positive cells in brain metastases and underline the importance of macrophages for metastatic progression of RCC.

Unfortunately, we were not able to study CCL2 expression in renal cancer because the CCL2 antibodies gave no reliable immunohistochemical staining results in formalin-fixed, paraffinembedded tumours (data not shown). Interestingly, our immunohistochemical analysis identified CCL7 expression in renal cancer cells as well as brain endothelial cells and confirmed a link between CCL7 tumour cell upregulation and increased metastatic capacity to the brain. CCL7 is one of the most pluripotent chemokines, acting on multiple cell types including monocytes, lymphocytes, eosinophils, basophils, dendritic cells and natural killer cells. There is limited knowledge about CCL7 expression in the brain. CCL7 is closely related to CCL2, which is one of the most commonly expressed chemokines in the CNS during inflammation. It is expressed in the perivascular space and brain parenchyma and is involved in recruitment of monocytes/macrophages, memory $\mathrm{T}$ cells and natural killer cells (Van Damme et al, 2004). Elevated CCL2 expression was linked to metastasis through the recruitment of monocytes/macrophages. CCL2 facilitates breast cancer metastasis to the lung and is associated with poor prognosis of breast, colorectal, prostate and cervix cancer because of metastatic progression (Zhang et al, 2010a, b; Soria et al, 2011). It is thus tempting to speculate that similar to CCL2, tumour-cell-derived CCL7 also attracts CCR2-positive cells of monocyte origin to facilitate brain metastasis via increased vascular permeability.

In summary, our results are relevant for the understanding of the metastatic pathways in RCC and the treatment effects of antiangiogenic therapies in RCC patients. Antimetastatic drugs have different potential targets, including myeloid-derived monocytes/macrophages, chemokines and their receptors (Qian et al, 2011). The exact mechanisms by which CCR2-positive TAMs promote tumour progression, migration and metastasis in RCC require extensive future examinations for better understanding.

\section{ACKNOWLEDGEMENTS}

The study was supported by the Swiss National Science Foundation (3238BO-103145) Cancer League Zurich and Swiss Cancer
Research to HM and the Helmholtz foundation, an ERC Starting grant (LiverCancerMechanism) and the Helmholtz Alliance preclinical comprehensive cancer centre (PCCC) to $\mathrm{MH}$.

\section{REFERENCES}

Andre F, Cabioglu N, Assi H, Sabourin JC, Delaloge S, Sahin A, Broglio K, Spano JP, Combadiere C, Bucana C, Soria JC, Cristofanilli M (2006) Expression of chemokine receptors predicts the site of metastatic relapse in patients with axillary node positive primary breast cancer. Ann Oncol 17(6): 945-951.

Bianchi M, Sun M, Jeldres C, Shariat SF, Trinh QD, Briganti A, Tian Z, Schmitges J, Graefen M, Perrotte P, Menon M, Montorsi F, Karakiewicz PI (2012) Distribution of metastatic sites in renal cell carcinoma: a population-based analysis. Ann Oncol 23(4): 973-980.

Bubendorf L, Schopfer A, Wagner U, Sauter G, Moch H, Willi N, Gasser TC, Mihatsch MJ (2000) Metastatic patterns of prostate cancer: an autopsy study of 1,589 patients. Hum Pathol 31(5): 578-583.

Colotta F, Allavena P, Sica A, Garlanda C, Mantovani A (2009) Cancer-related inflammation, the seventh hallmark of cancer: links to genetic instability. Carcinogenesis 30(7): 1073-1081.

Culine S, Bekradda M, Kramar A, Rey A, Escudier B, Droz JP (1998) Prognostic factors for survival in patients with brain metastases from renal cell carcinoma. Cancer 83(12): 2548-2553.

D'Alterio C, Cindolo L, Portella L, Polimeno M, Consales C, Riccio A, Cioffi M, Franco R, Chiodini P, Carteni G, Mirone V, Longo N, Marra L, Perdona S, Claudio L, Mascolo M, Staibano S, Falsaperla M, Puglisi M, Martignoni G, Ficarra V, Castello G, Scala S (2010a) Differential role of CD133 and CXCR4 in renal cell carcinoma. Cell Cycle 9(22): 4492-4500.

D’Alterio C, Consales C, Polimeno M, Franco R, Cindolo L, Portella L, Cioffi M, Calemma R, Marra L, Claudio L, Perdona S, Pignata S, Facchini G, Carteni G, Longo N, Pucci L, Ottaiano A, Costantini S, Castello G, Scala S (2010b) Concomitant CXCR4 and CXCR7 expression predicts poor prognosis in renal cancer. Curr Cancer Drug Targets 10(7): $772-781$.

Dahinden C, Ingold B, Wild P, Boysen G, Luu VD, Montani M, Kristiansen G, Sulser T, Buhlmann P, Moch H, Schraml P (2010) Mining tissue microarray data to uncover combinations of biomarker expression patterns that improve intermediate staging and grading of clear cell renal cell cancer. Clin Cancer Res 16(1): 88-98.

Dannenmann SR, Thielicke J, Stockli M, Matter C, von Boehmer L, Cecconi V, Hermanns T, Hefermehl L, Schraml P, Moch H, Knuth A, van den Broek M (2013) Tumor-associated macrophages subvert T-cell function and correlate with reduced survival in clear cell renal cell carcinoma. Oncoimmunology 2(3): e23562. 
de Vries NA, Beijnen JH, Boogerd W, van Tellingen O (2006) Blood-brain barrier and chemotherapeutic treatment of brain tumours. Expert Rev Neurother 6(8): 1199-1209.

Doolittle ND, Peereboom DM, Christoforidis GA, Hall WA, Palmieri D, Brock PR, Campbell KC, Dickey DT, Muldoon LL, O’Neill BP, Peterson DR, Pollock B, Soussain C, Smith Q, Tyson RM, Neuwelt EA (2007) Delivery of chemotherapy and antibodies across the blood-brain barrier and the role of chemoprotection, in primary and metastatic brain tumours: report of the Eleventh Annual Blood-Brain Barrier Consortium meeting. J Neurooncol 81(1): 81-91.

Eichler AF, Chung E, Kodack DP, Loeffler JS, Fukumura D, Jain RK (2011) The biology of brain metastases-translation to new therapies. Nat Rev Clin Oncol 8(6): 344-356.

Eichler AF, Loeffler JS (2007) Multidisciplinary management of brain metastases. Oncologist 12(7): 884-898.

Fischer T, Nagel F, Jacobs S, Stumm R, Schulz S (2008) Reassessment of CXCR4 chemokine receptor expression in human normal and neoplastic tissues using the novel rabbit monoclonal antibody UMB-2. PLoS One 3(12): e4069.

Gahan JC, Gosalbez M, Yates T, Young EE, Escudero DO, Chi A, Garcia-Roig M, Satyanarayana R, Soloway MS, Bird VG, Lokeshwar VB (2012) Chemokine and chemokine receptor expression in kidney tumors: molecular profiling of histological subtypes and association with metastasis. J Urol 187(3): 827-833.

Gay PC, Litchy WJ, Cascino TL (1987) Brain metastasis in hypernephroma. J Neurooncol 5(1): 51-56.

Glaves D, Huben RP, Weiss L (1988) Haematogenous dissemination of cells from human renal adenocarcinomas. Br J Cancer 57(1): 32-35.

Gouttefangeas C, Stenzl A, Stevanovic S, Rammensee HG (2007) Immunotherapy of renal cell carcinoma. Cancer Immunol Immunother 56(1): 117-128.

Helgason HH, Mallo HA, Droogendijk H, Haanen JG, van der Veldt AA, van den Eertwegh AJ, Boven E (2008) Brain metastases in patients with renal cell cancer receiving new targeted treatment. J Clin Oncol 26(1): 152-154.

Ingold B, Schraml P, Heppner FL, Moch H (2009) Homogeneous MGMT immunoreactivity correlates with an unmethylated MGMT promoter status in brain metastases of various solid tumors. PLoS One 4(3): e4775.

Ingold B, Wild PJ, Nocito A, Amin MB, Storz M, Heppner FL, Moch H (2008) Renal cell carcinoma marker reliably discriminates central nervous system haemangioblastoma from brain metastases of renal cell carcinoma. Histopathology 52(6): 674-681.

Laubli H, Borsig L (2010) Selectins promote tumor metastasis. Sem Cancer Biol 20(3): 169-177.

Lazarini F, Tham TN, Casanova P, Arenzana-Seisdedos F, Dubois-Dalcq M (2003) Role of the alpha-chemokine stromal cell-derived factor (SDF-1) in the developing and mature central nervous system. Glia 42(2): 139-148.

Lee BC, Lee TH, Avraham S, Avraham HK (2004) Involvement of the chemokine receptor CXCR4 and its ligand stromal cell-derived factor lalpha in breast cancer cell migration through human brain microvascular endothelial cells. Mol Cancer Res 2(6): 327-338.

Liang Z, Wu T, Lou H, Yu X, Taichman RS, Lau SK, Nie S, Umbreit J, Shim H (2004) Inhibition of breast cancer metastasis by selective synthetic polypeptide against CXCR4. Cancer Res 64(12): 4302-4308.

Massard C, Zonierek J, Gross-Goupil M, Fizazi K, Szczylik C, Escudier B (2010) Incidence of brain metastases in renal cell carcinoma treated with sorafenib. Ann Oncol 21(5): 1027-1031.

McCandless EE, Piccio L, Woerner BM, Schmidt RE, Rubin JB, Cross AH, Klein RS (2008a) Pathological expression of CXCL12 at the blood-brain barrier correlates with severity of multiple sclerosis. Am J Pathol 172(3): 799-808.

McCandless EE, Zhang B, Diamond MS, Klein RS (2008b) CXCR4 antagonism increases $\mathrm{T}$ cell trafficking in the central nervous system and improves survival from West Nile virus encephalitis. Proc Natl Acad Sci USA 105(32): 11270-11275.

Medioni J, Cojocarasu O, Belcaceres JL, Halimi P, Oudard S (2007) Complete cerebral response with sunitinib for metastatic renal cell carcinoma. Ann Oncol 18(7): 1282-1283.

Mildner A, Schmidt H, Nitsche M, Merkler D, Hanisch UK, Mack M, Heikenwalder M, Bruck W, Priller J, Prinz M (2007) Microglia in the adult brain arise from Ly-6ChiCCR $2+$ monocytes only under defined host conditions. Nat Neurosci 10(12): 1544-1553.
Mishra P, Banerjee D, Ben-Baruch A (2011) Chemokines at the crossroads of tumor-fibroblast interactions that promote malignancy. J Leukoc Biol 89(1): 31-39.

Motzer RJ, Bander NH, Nanus DM (1996) Renal-cell carcinoma. N Engl J Med 335(12): 865-875.

Muller A, Homey B, Soto H, Ge N, Catron D, Buchanan ME, McClanahan T, Murphy E, Yuan W, Wagner SN, Barrera JL, Mohar A, Verastegui E, Zlotnik A (2001) Involvement of chemokine receptors in breast cancer metastasis. Nature 410(6824): 50-56.

Nathoo N, Chahlavi A, Barnett GH, Toms SA (2005) Pathobiology of brain metastases. J Clin Pathol 58(3): 237-242.

Nathoo N, Toms SA, Barnett GH (2004) Metastases to the brain: current management perspectives. Expert Rev Neurother 4(4): 633-640.

Pan J, Burdick MD, Belperio JA, Xue YY, Gerard C, Sharma S, Dubinett SM, Strieter RM (2006a) CXCR3/CXCR3 ligand biological axis impairs RENCA tumor growth by a mechanism of immunoangiostasis. J Immunol 176(3): 1456-1464.

Pan J, Mestas J, Burdick MD, Phillips RJ, Thomas GV, Reckamp K, Belperio JA, Strieter RM (2006b) Stromal derived factor-1 (SDF-1/CXCL12) and CXCR4 in renal cell carcinoma metastasis. Mol Cancer 5: 56.

Phillips RJ, Burdick MD, Lutz M, Belperio JA, Keane MP, Strieter RM (2003) The stromal derived factor-1/CXCL12-CXC chemokine receptor 4 biological axis in non-small cell lung cancer metastases. Am J Respir Crit Care Med 167(12): 1676-1686.

Qian BZ, Li J, Zhang H, Kitamura T, Zhang J, Campion LR, Kaiser EA, Snyder LA, Pollard JW (2011) CCL2 recruits inflammatory monocytes to facilitate breast-tumour metastasis. Nature 475(7355): 222-225.

Salmaggi A, Maderna E, Calatozzolo C, Gaviani P, Canazza A, Milanesi I, Silvani A, DiMeco F, Carbone A, Pollo B (2009) CXCL12, CXCR4 and CXCR7 expression in brain metastases. Cancer Biol Ther 8(17): $1608-1614$.

Schouten LJ, Rutten J, Huveneers HA, Twijnstra A (2002) Incidence of brain metastases in a cohort of patients with carcinoma of the breast, colon, kidney, and lung and melanoma. Cancer 94(10): 2698-2705.

Shuch B, La Rochelle JC, Klatte T, Riggs SB, Liu W, Kabbinavar FF, Pantuck AJ, Belldegrun AS (2008) Brain metastasis from renal cell carcinoma: presentation, recurrence, and survival. Cancer 113(7): 1641-1648.

Soria G, Ofri-Shahak M, Haas I, Yaal-Hahoshen N, Leider-Trejo L, Leibovich-Rivkin T, Weitzenfeld P, Meshel T, Shabtai E, Gutman M, Ben-Baruch A (2011) Inflammatory mediators in breast cancer: coordinated expression of TNFalpha \& IL-1beta with CCL2 \& CCL5 and effects on epithelial-to-mesenchymal transition. BMC Cancer 11: 130 .

Staller P, Sulitkova J, Lisztwan J, Moch H, Oakeley EJ, Krek W (2003) Chemokine receptor CXCR4 downregulated by von Hippel-Lindau tumour suppressor pVHL. Nature 425(6955): 307-311.

Stamatovic SM, Shakui P, Keep RF, Moore BB, Kunkel SL, Van Rooijen N, Andjelkovic AV (2005) Monocyte chemoattractant protein-1 regulation of blood-brain barrier permeability. J Cereb Blood Flow Metab 25(5): 593-606.

Thibault F, Billemont B, Rixe O (2008) Regression of brain metastases of renal cell carcinoma with antiangiogenic therapy. J Neurooncol 86(2): 243-244.

Van Damme J, Struyf S, Opdenakker G (2004) Chemokine-protease interactions in cancer. Semin Cancer Biol 14(3): 201-208.

Wang X, Fujita M, Prado R, Tousson A, Hsu HC, Schottelius A, Kelly DR, Yang PA, Wu Q, Chen J, Xu H, Elmets CA, Mountz JD, Edwards 3rd CK (2010) Visualizing CD4 T-cell migration into inflamed skin and its inhibition by CCR4/CCR10 blockades using in vivo imaging model. Br J Dermatol 162(3): 487-496.

Weiss L, Harlos JP, Torhorst J, Gunthard B, Hartveit F, Svendsen E, Huang WL, Grundmann E, Eder M, Zwicknagl M (1988) Metastatic patterns of renal carcinoma: an analysis of 687 necropsies. J Cancer Res Clin Oncol 114(6): 605-612.

Wolf MJ, Hoos A, Bauer J, Boettcher S, Knust M, Weber A, Simonavicius N, Schneider C, Lang M, Sturzl M, Croner RS, Konrad A, Manz MG, Moch H, Aguzzi A, van Loo G, Pasparakis M, Prinz M, Borsig L, Heikenwalder M (2012) Endothelial CCR2 signaling induced by colon carcinoma cells enables extravasation via the JAK2-Stat5 and p38MAPK pathway. Cancer Cell 22(1): 91-105. 
Yoshidome H, Kohno H, Shida T, Kimura F, Shimizu H, Ohtsuka M, Nakatani Y, Miyazaki M (2009) Significance of monocyte chemoattractant protein-1 in angiogenesis and survival in colorectal liver metastases. Int J Oncol 34(4): 923-930.

Zhang J, Patel L, Pienta KJ (2010a) CC chemokine ligand 2 (CCL2) promotes prostate cancer tumorigenesis and metastasis. Cytokine Growth Factor Rev 21(1): 41-48.

Zhang J, Patel L, Pienta KJ (2010b) Targeting chemokine (C-C motif) ligand 2 (CCL2) as an example of translation of cancer molecular biology to the clinic. Prog Mol Biol Transl Sci 95: 31-53.
Zijlmans HJ, Fleuren GJ, Baelde HJ, Eilers PH, Kenter GG, Gorter A (2006) The absence of CCL2 expression in cervical carcinoma is associated with increased survival and loss of heterozygosity at 17q11.2. J Pathol 208(4): 507-517.

This work is published under the standard license to publish agreement. After 12 months the work will become freely available and the license terms will switch to a Creative Commons AttributionNonCommercial-Share Alike 3.0 Unported License. 